IPNO-DRE $93-30$

\title{
VECTOR MESON PRODUCTION AT CERN
}

C. Gerschel

Institut de Physique Nucléaire, 91406 Orsay Cedex, France 


\title{
VECTOR MESON PRODUCTION AT CERN
}

\author{
C. Gerschel \\ Institut de Physique Nucléaire, \\ 91406 Orsay Cedex, France
}

Invited talk at the

NATO ADVANCED STUDY INSTITUTE

ON HOT AND DENSE NUCLEAR MATTER

(September 26 - October 9, 1993)

Bodrum, Turquie 


\title{
VECTOR MESON PRODUCTION AT CERN
}

\author{
C. Gerschel \\ Institut de Physique Nucléaire \\ F-91406 ORSAY CEDEX
}

\section{INTRODUCTION}

Since the first oxygen beams at $200 \mathrm{GeV} /$ nucleon which were operated at CERN in 1986, an extensive experimental effort has been devoted to the study of the different proposed signatures of the formation of a quark-gluon plasma (QGP) ${ }^{1}$. At the same time, the obtained results have triggered a considerable amount of theoretical work leading to somewhat controversial interpretations.

Vector meson study gives the opportunity of having insight into two extensively debated signals : charmonium state and strangeness production. In the spirit of this school, I will remind the underlying basic physics, summarize the experimental results and try to discuss the interpretations available on the market at the present time.

\section{CHARMONIUM STATE PRODUCTION}

Following the original idea of Matsui and Satz ${ }^{2}$, charmonium state production should be suppressed in a QGP because of strong colour screening. This will appear when, at the characteristic formation time $\tau_{0}$ of the $\mathrm{J} / \psi$, the initial ce $\overline{\mathrm{c}}$ pair, from which $\mathrm{J} / \psi$ is issued, is still in the plasma in space or time. The suppression will thus be stronger at low transverse momentum $\mathrm{pT}^{3}$. It will also be stronger for $\psi^{\prime}$ than for $\mathrm{J} / \psi^{4}$. All this has been widely discussed in the previous years as described in the quoted references.

\section{$\mathrm{J} / \Psi$ Production}

$\mathrm{J} / \Psi$ production has been studied by the NA38 collaboration . It has been compared to that of dimuons in the mass continuum with an invariant mass $\mathbf{M}_{\mu \mu}$ between 1.7 and 2.7 $\mathrm{GeV} / \mathrm{c}^{2}$. The main results obtained are summarized thereafter :

- The ratio of the cross sections $B \sigma_{\mathrm{J} / \psi} / \sigma_{\text {cont }}$ for $\mathrm{J} / \psi$ and continuum formation decreases when increasing the neutral transverse energy $E_{0 T}$ of the collision ${ }^{5} . \mathrm{B}$ is the branching ratio for the decay of a $\mathrm{J} / \psi$ into a muon pair. Fig. 1 gives the more recent results of the NA38 experiment in the case of SU collisions ${ }^{6}$.

- It is the energy density which is the relevant parameter to relate the ratio $B \sigma_{\mathrm{J}} / \psi / \sigma_{\mathrm{cont}}$ for 


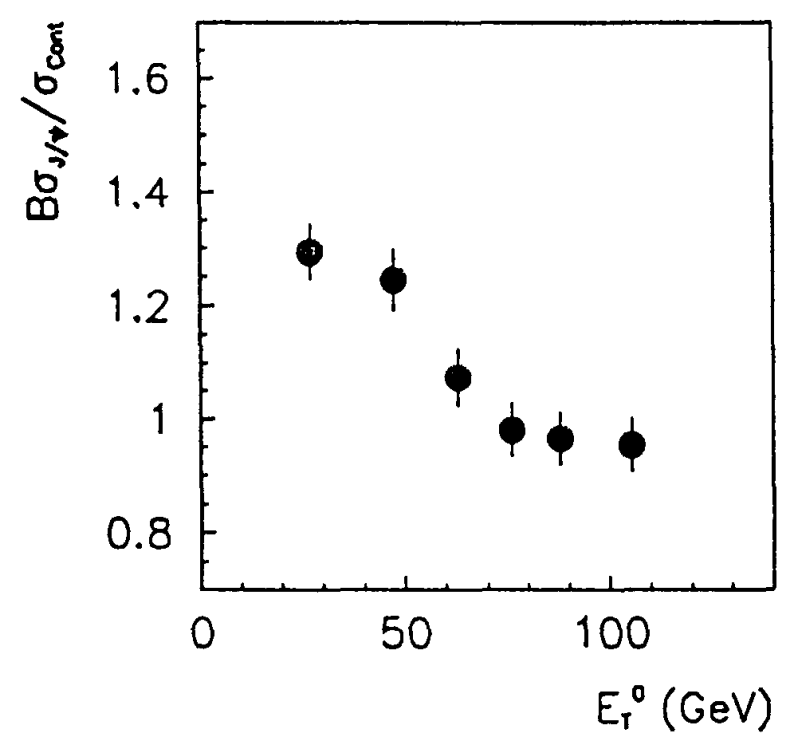

Fig.1 : Ratio of the production cross-sections for $\mathrm{J} / \psi$ and continuum dimuons for $S U$ as a function of the neutral transverse energies $E_{0} T$.

different target-projectile combinations (fig.2). The energy density $\varepsilon$ is defined according to the Björken estimate ${ }^{7} \varepsilon=3 \mathrm{E}_{\mathrm{oT}} / \mathrm{A}_{\perp} \tau_{0} \Delta \mathrm{y}$ where $\Delta \mathrm{y}=2.4$ is the rapidity range in which the $\pi^{0}$

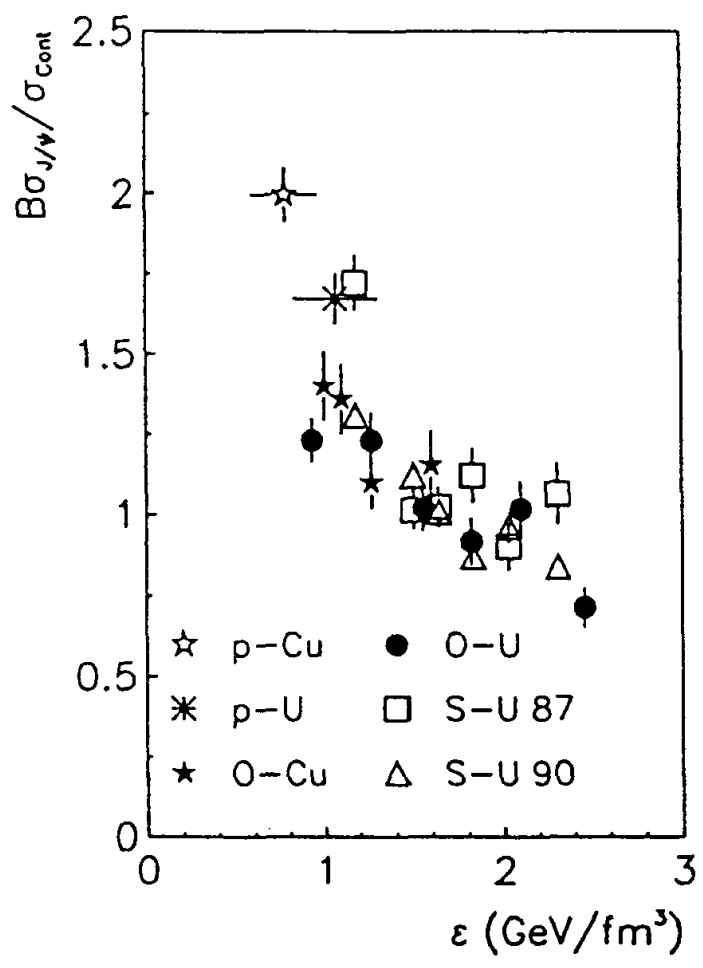

Fig. 2 : Ratio of the production cross-sections for $\mathrm{J} / \psi$ and continuum dimuons as a function of the energy density.

are measured, $A_{\perp}$ is the transverse overlap area of the collision at the corresponding $E_{0 T}$ value and at the formation time $\tau_{0}$ which is taken equal to $1 \mathrm{fm} / \mathrm{c} . A_{\perp}$ is deduced from the 
impact parameter obtained from a modelisation of the $E_{o T}$ distribution 8 . In the case of $p A$ collisions where no selection on the impact parameter is performed, $A_{\perp}$ is taken equal to $\pi r_{0}{ }^{2}$ with $r_{0}=1.1 \pm 0.1 \mathrm{fm}$. The use of the Björken estimate is somewhat incorrect at CERN energies where the transparency regime is not yet reached. However, it provides a reasonable relative scale and, in that sense, is also applied to such a small projectile as the proton.

- The decrease of $B \sigma_{\mathrm{J}} / \psi / \sigma_{\text {cont }}$ is due to a $\mathrm{J} / \psi$ suppression and not a continuum enhancement. The cross-section of the continuum has been measured for different combinations of target $A$ and projectile $B$. It is found to depend linearly on their atomic numbers : $\sigma_{\text {cont }}=\sigma_{\mathrm{o}}(\mathrm{AB}) \alpha_{\text {cont }}$ with $\alpha_{\text {cont }}=1.01 \pm 0.04{ }^{9}$. And it is indeed the $\mathrm{J} / \psi$ production which is suppressed. The corresponding $\alpha$ value for $\mathrm{J} / \psi$ is $\alpha_{\psi}=0.91 \pm 0.049$.

- The $\mathrm{J} / \psi$ suppression is stronger at low $\mathrm{p}_{\mathrm{T}}{ }^{10}$ and more so when the transverse energy increases. The latest results of NA38 6 are displayed on fig.3a.
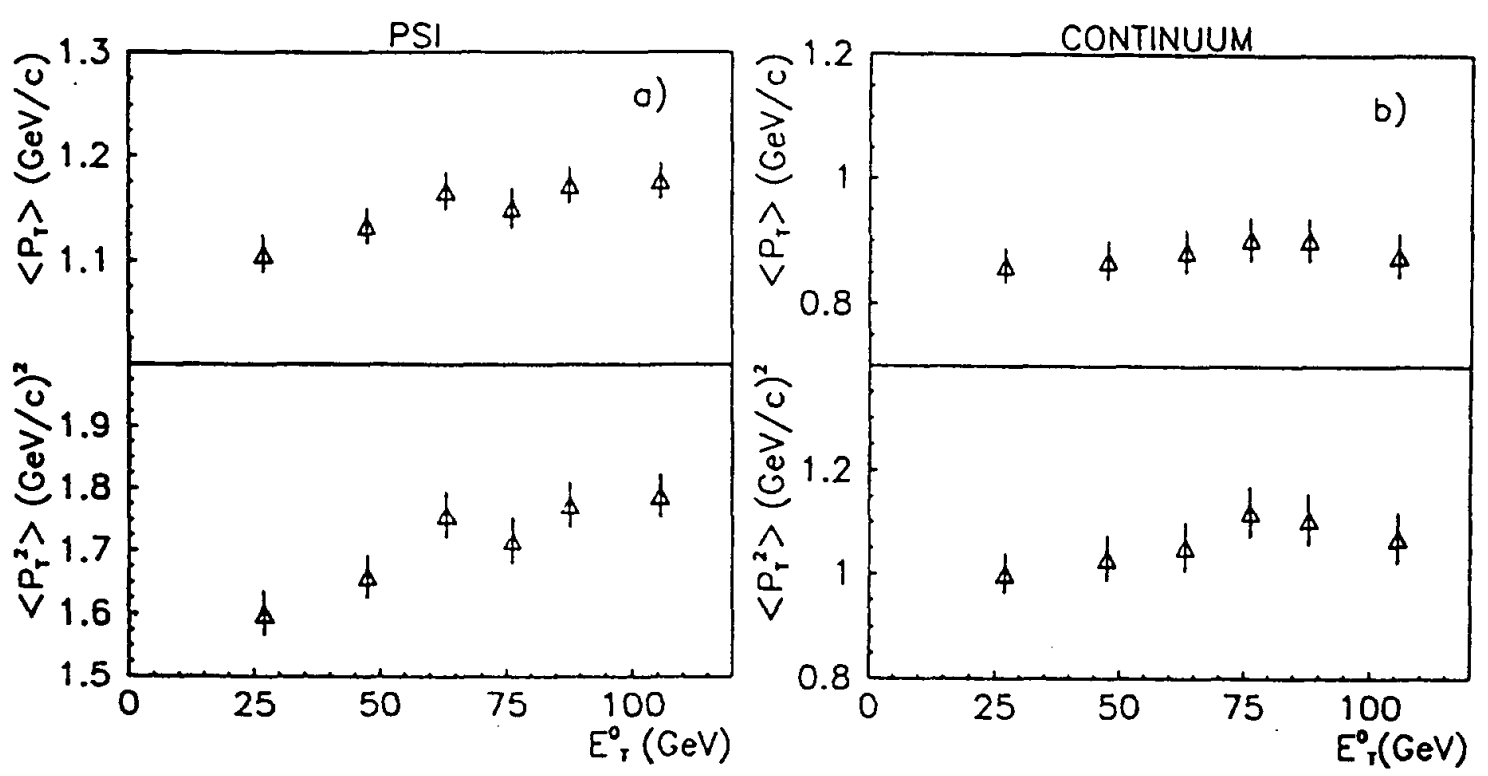

Fig. $3:<\mathrm{pT}>$ and $<\mathrm{pT}^{2}>$ as a function of the neutral transverse energy a) for $\mathrm{J} / \psi \mathrm{b}$ ) for continuum dimuons.

- The effects of nuclear matter on the $\mathrm{p}_{\mathrm{T}}$ distribution are stronger for $\mathrm{J} / \Psi$ than for continuum (fig.3b). One possibility of characterizing the effect of nuclear matter is to define the shift $\triangle \mathrm{pT}^{2}$ of the mean value of $\mathrm{pT}^{2}$ for a $\mathrm{AB}$ collision at a given $\mathrm{E}_{\mathrm{O}} \mathrm{T}$ as compared to that of a $\mathrm{pp}$ collision : $\Delta \mathrm{p}_{\mathrm{T}}^{2}\left(\mathrm{E}_{\mathrm{oT}}\right)=\left\langle\mathrm{pT}^{2}\right\rangle\left(\mathrm{E}_{\mathrm{oT}}\right)-\left\langle\mathrm{pT}^{2}\right\rangle_{\mathrm{pp}}$

It is found from fig. 2 that $\Delta \mathrm{pT}^{2}(\mathrm{~J} / \psi)=(2.5 \pm 0.5) \Delta \mathrm{pT}^{2}$ (cont) The values of $\left\langle\mathrm{pT}^{2}>_{\mathrm{pp}}\right.$ for $\mathrm{J} / \mathrm{\psi}$ and for continuum are taken from NA38 11 .

\section{$\Psi$ ' Production}

New results concerning $\psi^{\prime}$ production have been presented at QM 93 by the NA38 collaboration 12 . They show a stronger suppression for $\psi^{\prime}$ than for $J / \psi$. They can be summarized as follows :

- The ratio of the production cross-sections decreases when going from $\mathrm{pW}$ and $\mathrm{pU}$ to $\mathrm{SU}$ collisions (table $\mathrm{I}$ ).

- This ratio decreases also with the neutral transverse energy of the collision $E_{o T}$ (fig.4) in the case of the SU system. 
Table I : Ratio of the production cross-sections for different systems. Only statistical errors are shown.

\begin{tabular}{cc}
\hline System & $\mathrm{B} \sigma \psi^{\circ} / \mathrm{B} \sigma \mathrm{J} / \psi(\%)$ \\
\hline p-W & $1.77 \pm 0.22$ \\
p-U & $2.01 \pm 0.44$ \\
S-U & $1.07 \pm 0.09$ \\
\hline
\end{tabular}

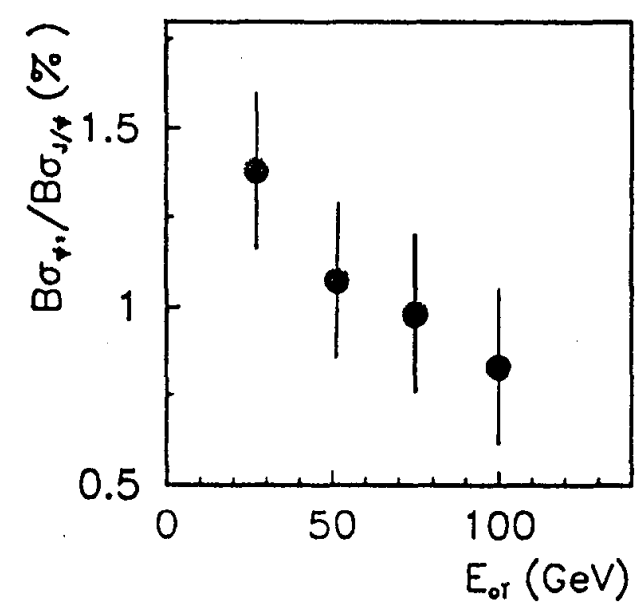

Fig.4 : Ratio of the production cross-sections for $\psi^{\prime}$ and $J / \psi$ for $S U$ as a function of the neutral transverse energy of the collision.

\section{Interpretation}

All these results are expected in the case of a QGP formation. However alternative explanations have been proposed which take care of the complicated environment of a nuclear collision. These effects are present in a pA as compared to pp collisions. They have been extrapolated to BA collisions and should be taken into account before to extract a genuine signal characteristic of a QGP formation in $A B$ collisions.

Informations which are available for $\mathrm{J} / \Psi$ production deal with the integrated crosssections for $\mathrm{pA}, \pi \mathrm{A} \overline{\mathrm{p}} \mathrm{A}$ and $\gamma \mathrm{A}$ collisions, the $\mathrm{pT}$ and $\mathrm{xF}_{\mathrm{F}}$ - dependences $13,14,15$. The $\mathrm{x}_{\mathrm{F}}$ dependence will not be discussed here and the reader is refered to the paper by E. Quack in these proceedings.

The $\mathrm{p}_{\mathrm{T}}$ - dependence in the case of particle-A collisions is usually interpreted in terms of multiple scattering in the initial state of the gluon of the projectile on the nucleons of the target 16,17 . This process has been extrapolated to BA collisions and there is at the present time a general consensus that the $\mathrm{p}_{\mathrm{T}}$ - dependence of $\mathrm{J} / \psi$ production observed by the NA38 experiment is well reproduced by initial state interactions ${ }^{11}$. It has been stressed ${ }^{17}$ that the effect of nuclear matter should be $9 / 4$ times stronger for $J / \psi$ (initial scattering of gluons) than for dimuons in the mass continuum (initial scattering of quarks) and indeed the value of $2.5 \pm$ 0.5 obtained from the new data of NA38 strengthens this hypothesis. Using this interpretation, at a given $\mathrm{E}_{\mathrm{oT}} \mathrm{T}$, the shift $\Delta \mathrm{pT}^{2}$ of $<\mathrm{pT}^{2}>$ can provide an "experimental" value of the mean length $\mathrm{L}_{\mathrm{i}}$ of nuclear matter available in the initial state at this $\mathrm{E}_{\mathrm{O}} \mathrm{T}^{15}$. This length has to be compared to the geometrical dimension of the nuclei, and any value of $\mathrm{L}_{\mathrm{j}}$ larger than 
the radius of the nuclei for central collisions will sign the appearence of new physics depending on $\mathrm{PT}$. This has to be checked for the forthcoming $\mathrm{Pb}-\mathrm{Pb}$ experiments.

The understanding of the cross-sections is much more debated and controversial. In terms of the conventional parametrization $\sigma_{(\text {part } A)}=\sigma_{(\text {part } N)} A^{\alpha}, \alpha$ is found to be of the order of 0.91 but with large fluctuations from one experiment to another one. It has been shown 15 that, in fact, the length $\mathrm{L}_{\mathrm{f}}$ of nuclear matter in the final state is a more adequate parameter to describe the cross-sections and using $\mathrm{L}_{\mathrm{f}}$, no discontinuity is observed between part-A, whatever the nature of the particle, and BA collisions (fig.5). Such a behavior can be

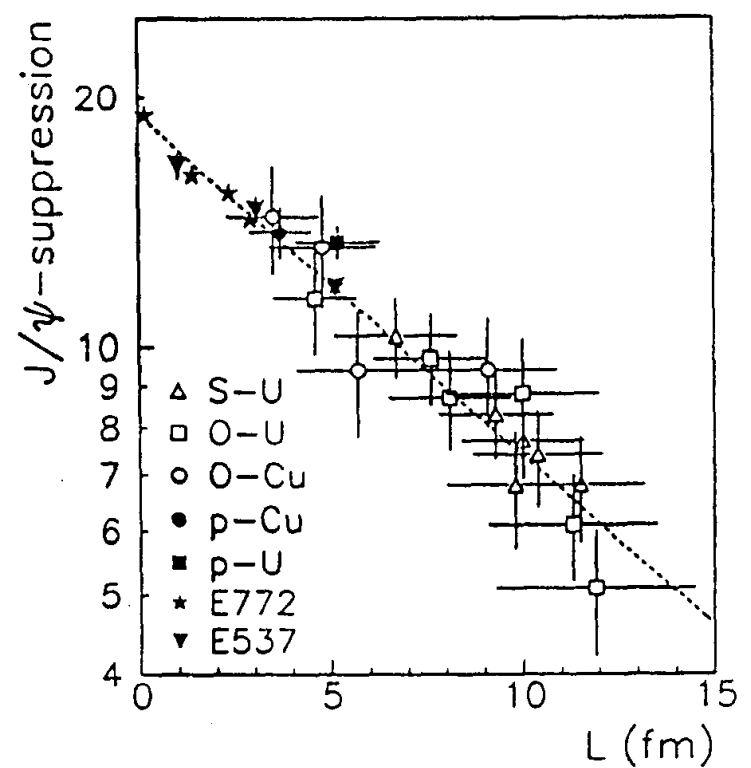

Fig.5 : Suppression of J/ $\psi$ as function of the length of nuclear matter in the final state. For BA collisions, it is B $\sigma_{\mathrm{J}} / \psi / \sigma_{\mathrm{cont}}$ which is plotted. An exponential fit to the data is shown. For the experiments E772 and E537, it is the crosssections which are plotted. Their values are arbitrarily normalized so that the heavier target agrees with the exponential fit.

interpreted 15 in terms of nuclear absorption leading to the dissociation of $J / \psi$ into a pair of $D$ mesons. Absorption cross-sections are found similar for part-A (6.3 $\pm 0.3 \mathrm{mb})$ and BA collisions $(6.9 \pm 1.0 \mathrm{mb})$. However, it is not clear which object interacts with the nucleus. It has been objected 18 that, taking into account the Lorentz boost of $\mathrm{J} / \psi$ as compared to nuclear matter, the final resonance is formed far away from the nucleus in the case of particle-A and it should be only a point-like object which crosses the nucleus. Other effects are thus proposed to account for the cross-sections. Final state interactions of $J / \psi$ with comovers ${ }^{18,19}$ has the same $L_{\mathbf{f}}$ dependence than nuclear absorption. They depend, of course, on the life-time of the hadron gas. And, as pointed out by Lesniak ${ }^{20}$, they are in contradiction with coherent $\mathrm{J} / \Psi$ production experiments ${ }^{21}$. In such a process, no comovers are produced, and indeed an absorption cross-section of $\sim 6 \mathrm{mb}$ is requested to explain the data. This stresses the leading influence of nuclear matter.

A reduction of the production cross-section could also be observed if some shadowing of the gluon densities in the nuclei is present ${ }^{22}$. This initial state effect should exist as observed for quark densities 23 but there is no clear indication about its size. Its proposed parametrization 22 is in contradiction with the observed $L_{f}$ scaling.

The interpretation of $J / \psi$ suppression is thus rather tricky. However, some recent developments seem promising, thanks to $\psi$ ' data. Contrary to $J / \psi, \psi$ ' seems to exhibit a different behavior between $\mathrm{pA}$ and $\mathrm{BA}$ collisions. The ratio $\mathrm{R}=\mathrm{B} \sigma_{\psi}, \mathrm{B} \sigma_{\mathrm{J} / \psi}$ looks constant 
for pA collisions ${ }^{12}$. The Fermilab experiment E772 13 has also found that $\alpha_{\psi}{ }^{\prime} \sim \alpha_{\psi}$ at $\left\langle\mathrm{x}_{\mathrm{f}}\right\rangle=\mathbf{0 . 3}$. This similar behavior of $\mathrm{J} / \psi$ and $\psi$ ' for $\mathrm{pA}$ collisions can be explained if it is the same object which crosses the nucleus in both cases.

$\mathrm{J} / \psi$ and $\psi^{\prime}$ productions are considered as a three step process : i) production of a $\mathrm{c} \overline{\mathrm{c}}$ point-like colour octet state by gluon fusion ii) emission or absorption of a third gluon leading to colour neutrality iii) evolution towards the fully grown resonance state. It has been proposed that the A-dependence of the cross-sections ${ }^{25}$ and the $\mathrm{x}_{\mathrm{f}}$-behavior ${ }^{26}$ are due to the interaction of the colour octet state with the target nucleus. Of course, the final effect will depend strongly on the time of emission of the third gluon and it is not yet clear whether this can account for the $\mathrm{L}_{\mathrm{f}}$ scaling for $\mathrm{J} / \psi$ observed at the different center of mass energies. More work needs to be done but this offers an interesting issue. In the case of BA collisions, $\mathrm{J} / \psi$ formation times are shorter due to smaller Lorentz $\gamma$ factor and it is the final size resonances which interact with the matter. The decrease of the ratio $R$ in the case of $S U$ could then be simply explained by the interactionof $J / \psi$ and $\psi$ ' with nuclear matter. On fig.6, $\mathrm{R}$ is plotted as a function of $L_{\mathrm{f}}$. The values for $\mathrm{pA}$ collisions are corrected both for the $\sqrt{\mathrm{s}}$ dependence of the cross-sections ${ }^{26}$ and for the feeding of the $\psi$ from the $\psi$ ' and the $\chi$, using the values measured for $\mathrm{pLi}$ at $300 \mathrm{GeV} / \mathrm{c}^{27}$. One observes on the figure the constant value of $\mathrm{R}$ for $\mathrm{pA}$ collisions ant its decrease for SU. The exponential fit for S-U data gives a ratio of the absorption cross sections between the $\psi$ ' and the $\psi$ of $2.1 \pm 0.6$. If the absorption crosssections scale like the mean squared radius of the resonances ${ }^{28}$, a ratio 2.3 is expected, taking into account the fact that $40 \%$ of the observed $\psi$ are fed from direct $\chi$ or $\psi^{\prime} 27$. More precise results would be welcomed.

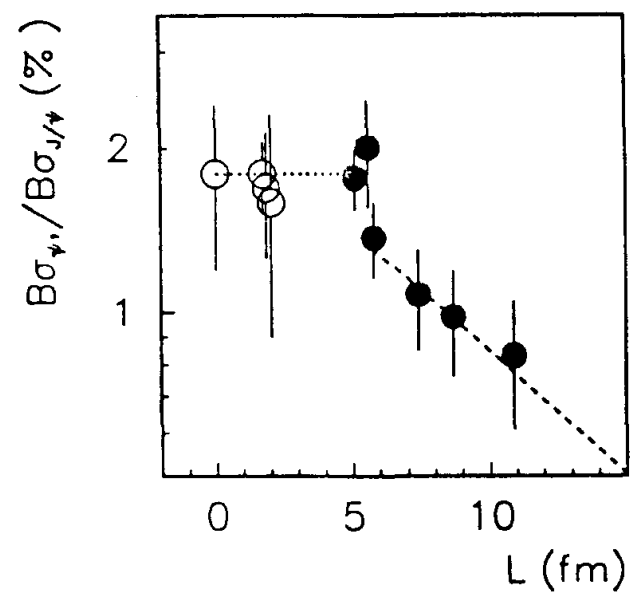

Fig.6 : Ratio of the production cross-sections for $\psi^{\prime}$ and $\mathrm{J} / \psi$ as a function of the length of nuclear matter in the final state. The black dotes correspond to NA38 results, the open dots to other pA experiments ${ }^{12}$.

\section{Conclusion}

No definite explanation can be given at the present time about $\psi$ and $\psi$ ' suppression in heavy ion collision induced by $\mathrm{O}$ and $\mathrm{S}$ projectiles. $\mathrm{J} / \psi$ and $\psi^{\prime}$ behave differently in comparison with $\mathrm{pA}$ collisions. If not unambiguously characteristic of the existence of a QGP, this fact should improve our knowledge of time scales involved in the process. A good reference is now available for any new physics appearing in the forthcoming $\mathrm{Pb}-\mathrm{Pb}$ experiments. 


\section{PRODUCTION OF LOW MASS VECTOR MESONS}

The $\phi$ meson, composed of a strange and anti-strange quark, is the lightest vector meson with a hidden flavor. Its behavior can be compared to that of the $\rho$ and $\omega$ which are resonances of the light quark-antiquark systems and thus give access to strangeness content of heavy-ion collisions. It had indeed been proposed ${ }^{29}$ that the creation of a QGP would result in an enhanced production of strange quarks. The $\phi$ could be an excellent probe of $\mathrm{QGP}^{30}$ as its production and decay in pp collisions is suppressed by the Okubo-Zweig-Lizuka (OZI) rule 31 , which requires that transitions take place by means of connected quark diagrams. In case of QGP formation, the lack of the OZI suppression in addition to the abundance of strange quarks would lead to an enhanced production of $\phi$ meson.

\section{Experimental results}

Low mass vector mesons have been studied both by the NA38 and Helios/ 3 collaborations, through their decay channels into two muons. Due to the small mass difference between the $\rho$ and the $\omega(13 \mathrm{MeV})$ and to the finite mass resolution of the spectrometers, $\rho$ and $\omega$ contributions are not experimentally discriminated and the $\phi$ production is compared to the sum $\rho+\omega$.

The main results are the following:

- NA38 has shown that the ratio of the cross-sections $B_{\phi} \sigma_{\phi} /\left(B_{\rho} \sigma_{\rho}+B_{\omega} \sigma_{\omega}\right)$ increases from $\mathrm{pW}$ to $S U$, and with the neutral transverse energy $E_{\mathrm{OT}}$ in the case of $S^{32}$ (fig.7). Similar results have been obtained by Helios $/ 3$ for $\mathrm{pW}$ and $\mathrm{SW}$ 33. An analogous increase was also observed previously at high $\mathrm{p}_{\mathrm{T}}$ by the NA38 collaboration in the case of $\mathrm{O}-\mathrm{U}^{34}$.The overall increase is of about a factor two.

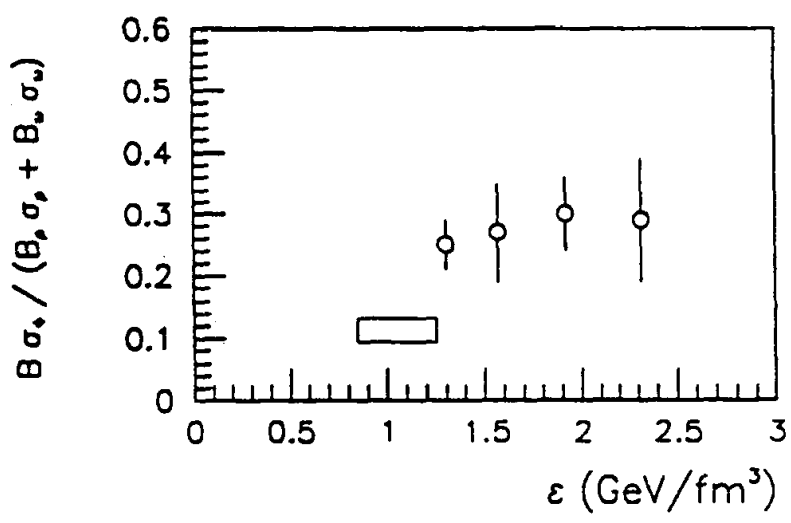

Fig.7 : Ratio of the production cross-sections for $\phi$ and $p+\omega$ from NA38 as a function of the energy density. Open dots : SU. Rectangle : pW.

Table II : Ratio of the production cross-sections for $\phi$ and $\rho+\omega$ from Helios/3. Comparison between different PT-bins.

\begin{tabular}{ccc}
\hline$\phi /(\rho+\omega)$ & $\mathrm{p}+\mathrm{W}$ & $\mathrm{S}+\mathrm{W}$ \\
\hline $\mathrm{pT} \leq 0.35 / \mathrm{pT}_{\mathrm{T}}>0.6$ & $0.83 \pm 0.10$ & $0.95 \pm 0.17$ \\
$0.35 \leq \mathrm{pT}<0.6 / \mathrm{pT}>0.6$ & $0.98 \pm 0.11$ & $0.70 \pm 0.11$ \\
\hline
\end{tabular}


- The increase of this ratio does not seem to depend on pT 32,33 as observed by NA38 (fig.8) and by Helios/3 (table II).

- As shown by NA38, the increase of this ratio seems to be due more to a $\phi$ enhancement than to a $\rho+\omega$ suppression 32 . From the A-dependence of the cross-sections between $p W$ and $\mathrm{SU}$, it is possible to deduce the $\alpha$ parameter. It is found that, for $\mathrm{pT}>0.6 \mathrm{GeV} / \mathrm{c}$, $\alpha_{\phi}=1.24 \pm 0.03 \pm 0.08$ while $\alpha_{p+\omega}=1.01 \pm 0.02 \pm 0.13$.

- There is a discontinuity between pA and BA collisions. From NA38 cross-sections, we get $\alpha_{\phi}-\alpha_{\rho+\omega}=0.23 \pm 0.04 \pm 0.0932$. This has to be compared to the value obtained for pA collisions $\alpha_{\phi}-\alpha_{p+\omega}=0.05 \pm 0.0535$.
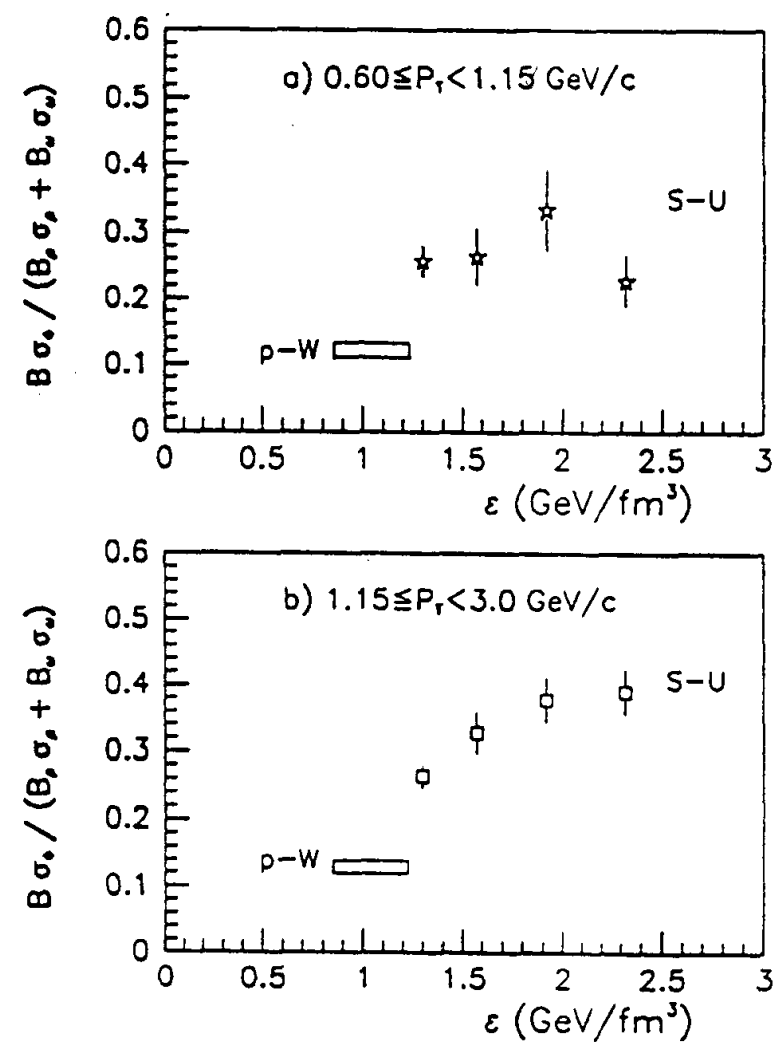

Fig.8 : Ratio of the production cross-sections for $\phi$ and $\rho+\omega$ from NA38 as a function of energy density, for two different pT bins.

\section{Interpretation}

As in the case of $\mathrm{J} / \psi$, these results are in agreement with what is expected if a QGP is formed 30,36 . However, strange particles can also be produced in hadronic reactions before the nuclear fireball reaches equilibrium 37,38 (see also the paper by $H$. Sorge in these proceedings). In RQMD calculations, the cascade leading to $\phi$ production has been studied in detail and shows an important contribution of meson-meson interactions over the whole rapidity range.

The $\phi$ enhancement must thus be considered in the general framework of strangeness enhancement and do not alone provide a reliable signature for the QGP.

\section{CONCLUSION}

Striking experimental effects have been observed both for $\mathrm{J} / \psi, \psi$ ' and $\phi$ production in heavy ion collisions. Great theoretical efforts have been made in view of a comparison 
with avalaible particle-A data. Unfortunately, the initial non ambiguousness of the possible signatures of a QGP has been progressively washed out and it is not clear today wether we deal with a new state of matter or not. As pointed out, in a provocative way, by Blaizot in the summary talk of Quark Matter 93 Conference, are we sensitive, at present CERN energies, to quark and gluon degrees of freedom in the observables we are looking at ? If the answer is yes, we have now a better knowledge of hadronic effects which is a prerequisite to recognize any new physics appearing in the forthcoming $\mathrm{Pb}-\mathrm{Pb}$ experiments.

\section{REFERENCES}

1. Proceedings of Quark Matter Conferences.

2. T. Matsui and H. Satz, Phys. Lett. B178: 416 (1986).

3. F. Karsch and R. Petronzio, Phys. Lett. B193 : 105 (1987) ; Z. Phys. C37 : 627 (1988).

4. F. Karsch et al., Z. Phys., C37 : 617 (1988).

C. Gerschel and J. Hüfner, Phys. Lett. B207 : 253 (1988).

5. C. Baglin et al., NA38 Collaboration, Phys. Lett. B220 : 471 (1989) ; B255 : 459 (1991).

C. Baglin et al., NA38 Collaboration, Phys. Lett. B251: 465 (1990).

O. Drapier, NA38 Collaboration, Nucl. Phys. A544 : 209c (1992).

6. R. Mandry, PhD Thesis, Lyon (1993).

7. J.D. Björken, Phys. Rev. D27 : 140 (1983).

8. C. Baglin et al., NA38 Collaboration, Phys. Lett. B251: 472 (1990).

9. C. Baglin et al., NA38 Collaboration, Phys. Lett. B270 : 105 (1991).

10. C. Baglin et al., NA38 Collaboration, Phys. Lett. B251: 465 (1990).

C. Baglin et al., NA38 Collaboration, Phys. Lett. B262 : 362 (1991).

11. C. Baglin et al., NA38 Collaboration, Phys. Lett. B268 : 453 (1991).

12. C. Lourenço, NA38 Collaboration, Proceedings of Quark Matter 93 Conference. B. Ronceux, NA38 Collaboration, Proceedings of Quark Matter 93 Conference.

13. D.M. Alde et al., Phys. Rev. Lett. $66: 133$ (1991).

14. S. Katsanevas et al., Phys.Rev. Lett. $60: 2121$ (1988).

15. C. Gerschel and J. Hüfner, Z. Phys. C56 : 171 (1992).

16. S. Gavin and M. Gyulassy, Phys. Lett. B214 : 241 (1988). J.P. Blaizot and J.Y. Ollitrault, Phys. Lett. B217 : 392 (1989).

17. J. Huefner, Y. Kurihara and H. Pirner, Phys. Lett. B215 : 218 (1988).

18. R. Vogt, Nucl. Phys. A544 : 615c (1992).

19. J. Ftanik, P. Lichard and J. Pisut, Phys. Lett. B207 : 194 (1988).

S. Gavin, M. Gyulassy and A. Jackson, Phys. Lett. B207 : 257 (1988).

R. Vogt et al., Phys. Lett. B207 : 263 (1988).

J.P. Blaizot and J.Y. Ollitrault, Phys. Rev. D39 : 232 (1989).

20. L. Lesniak, Phys. Lett. B302 : 140 (1993).

21. M.D. Sokoloff et al., Phys. Rev. Lett. $38: 263$ (1977)

P. Amaudruz et al., (NMC Coll.), Nucl. Phys. B371 : 553 (1992)

22. S. Gupta and H. Satz, Z. Phys. C51 : 209 (1991).

23. R. Seitz and A. Witzman (NMC Coll.) , Proceedings of the XXVIIIth Rencontre de Moriond (1993).

24. G. Piller, T. Mutsbauer and W. Weise, Nucl. Phys. A560 : 437 (1993).

25. D. Kharzeev and H. Satz, Z. Phys. C60 : 389 (1993).

26. P. Sonderegger, private communication.

27. L. Antoniazzi et al., Phys. Rev. Lett. $70: 383$ (1993).

28. B. Povh and J. Hüfner, Phys. Rev. Lett. 58 : 1612 (1987).

29. J. Rafelski and B. Muller, Phys. Rev. Lett. 48 : 1066 (1982). 
30. A. Shor, Phys. Rev. Lett. 54 : 1122 (1985).

31. S. Okubo, Phys. Rev. D16 : 2336 (1977).

32. NA38 Collaboration, R. Ferreira, Nucl. Phys. A544 : 497c (1992).

R. Ferreira, Ph.D Thesis, Lisbonne (1992).

33. Helios/3 Collab., M.A. Mazzoni, Nucl. Phys. A544 : 623c (1992)

R.J. Apsimon et al., Z. Phys. C53 : 581 (1992).

34. NA38 Collaboration, C. Baglin et al., Phys. Lett. B272 : 449 (1991).

35. W. Geist, Nucl. Phys. A525: 149c (1991).

36. H.W. Barz et al., Nucl. Phys. A484 : 661 (1988) ; Nucl. Phys. A525 : 435c (1991).

37. P. Koch et al., Z. Phys. C47: 477 (1990).

38. M. Berenguer et al., Proc. $9^{\text {th }}$ Winter Workshop on Nuclear Dynamics, Key West, USA (1993).

M. Berenguer, Ph. D Thesis, Francfort (1993). 